\title{
Breakthrough cases of COVID-19 in vaccinated United States Veterans with spinal cord injuries and disorders
}

(c) The Author(s), under exclusive licence to International Spinal Cord Society 2021

Spinal Cord (2021) 59:1132-1133; https://doi.org/10.1038/s41393021-00690-w

\section{TO THE EDITOR:}

While the currently authorized vaccines for coronavirus disease 2019 (COVID-19) are highly effective in the general population, little is known about the influence specific conditions may have on vaccine breakthrough. Our operational review of Veterans Health Administration (VHA) medical records pertaining to COVID-19 infections and vaccinations is the first to provide preliminary data on vaccine breakthrough in Veterans with spinal cord injuries and disorders $(\mathrm{SCl} / \mathrm{D})$.

The VHA SCI/D National Program Office maintains the VHA SCl/ D Registry, a database of Veterans within the VHA SCI/D System of Care. As of April 5, 2021, there were 17,777 living Veterans with $\mathrm{SCl} / \mathrm{D}$ identified in the registry. During the COVID-19 pandemic, the National Program Office developed monitoring reports for COVID-19 that include dates of infections and vaccinations [1]. For this operational review, cases with vaccination and infection dates consistent with vaccine breakthrough were validated by reviewing electronic medical records. Per CDC guidance we defined a breakthrough infection as a positive SARS-CoV-2 test 14 or more days after completing COVID-19 vaccination [2]. This review included veterans with both traumatic and nontraumatic etiology of $\mathrm{SCl} / \mathrm{D}$, but excluded veterans with multiple sclerosis and motor neuron diseases.

COVID-19 vaccine breakthrough was identified in 17 out of 8319 $(0.20 \%)$ of fully vaccinated Veterans with SCI/D as of April 20, 2021. Two additional cases were identified as false-positive cases and were excluded from analysis. Overall, 4161 individuals received the Pfizer-BioNTech vaccine series, 3991 received the Moderna vaccine series, and 167 individuals received the Johnson \& Johnson Janssen single vaccine. Eleven breakthrough cases occurred after the Pfizer-BioNTech vaccine series and six occurred after the Moderna vaccine series. No cases occurred following the Johnson \& Johnson Janssen vaccine. The median time from the final vaccine dose to positive COVID test in our data was 50 days (mean 64 days, ranged from 15-90 days). The positive COVID-19 tests occurred in a variety of settings including surveillance (5), pre-procedure testing (5), ER (5), and post-exposure (2). Of those diagnosed with COVID-19, 6 (35.3\%) were symptomatic and 11 $(64.7 \%)$ were asymptomatic. Of the six symptomatic cases, three were of mild severity, one was moderate, and two were severe based on CDC classification guidelines [3]. Three patients were hospitalized, and one patient died within 30 days of COVID-19 diagnosis. All cases occurred in male patients, with an average age of 69 years (range 31-88). Of 16 cases with neurological level recorded in the registry, ten $(63 \%)$ had tetraplegia, and six (37\%) had paraplegia. Living setting at time of diagnosis was home for 12 cases and skilled nursing facility (SNF) for five cases. Of importance, three asymptomatic cases occurred during an outbreak involving staff and patients at a single SNF.

As of April 20, 2021, the CDC reported 7157 vaccine breakthrough cases out of $87,000,000$ fully vaccinated individuals in the United States, resulting in a vaccine breakthrough rate of $0.0082 \%$ [2]. Although the breakthrough rate is 24 times lower than that seen in our review, the CDC data include few asymptomatic cases, whereas for the VA SCI/D population (which is subject to more frequent surveillance testing) asymptomatic cases made up the majority. A more appropriate comparison may be an investigation of breakthrough cases conducted by the CDC in residents and staff members from 78 SNF's in Illinois. Their analysis demonstrated 22 COVID-19 cases out of 14,765 vaccinated individuals, a rate of $0.15 \%$. There was a similar rate of asymptomatic infections (64\%) as in the Veteran $\mathrm{SCl} / \mathrm{D}$ population and a similarly low rate of hospitalizations related to COVID-19 (two out of the 22 cases), and one death [4]. These similarities suggest that the increased breakthrough rate seen in our review may, in part, be related to increased congregate living and higher rate of surveillance testing in individuals with $S C I / D$, rather than due to the $S C l / D$ itself. The CDC also recently investigated a COVID-19 outbreak in a single SNF in Kentucky, which demonstrated a high rate of COVID-19 breakthrough infection in the fully vaccinated SNF residents $(25 \%)$, although these were similarly predominantly asymptomatic infections (67\%) [5]. That investigation suggests reduced overall vaccine effectiveness during a widespread disease outbreak, but with protection against severe disease.

In summary, our preliminary review indicates a similar rate of COVID-19 Vaccine breakthrough in individuals with SCI/D compared to residents of SNFs in which frequent surveillance testing is occurring. Given the uncertainty about the completeness of the CDC data for the general population, and our relatively few symptomatic COVID-19 breakthrough cases, we are unable to draw definitive conclusions about the effectiveness of the vaccine in preventing COVID-19 in the SCl/D population. Our findings support ongoing efforts to minimize COVID-19 exposure and spread in fully vaccinated individuals with $S C I / D$, particularly in congregate living settings at high risk of COVID-19 outbreak. However, the predominance of asymptomatic cases in vaccinated individuals with SCI/D highlights the importance of vaccination in decreasing morbidity and mortality associated with COVID-19 infection in the $\mathrm{SCl} / \mathrm{D}$ population. 
Kendl M. Sankary $\mathbb{D}^{1,2 \bowtie}$, Jennifer L. Sippel ${ }^{3}$, Adam C. Eberhart ${ }^{4}$ and Stephen P. Burns (iD) ${ }^{1,2}$ ${ }^{1}$ Spinal Cord Injury Service, VA Puget Sound Health Care System, Seattle, WA, USA. ${ }^{2}$ Department of Rehabilitation Medicine, University of Washington School of Medicine, Seattle, WA, USA. ${ }^{3}$ Spinal Cord

Injuries and Disorders National Program Office, Veterans Health Administration, Washington, DC, USA. ${ }^{4}$ Spinal Cord Injury Service, VA St. Louis Health Care System, St. Louis, MO, USA.

凶email: ksankary@uw.edu

\section{REFERENCES}

1. Burns SP, Eberhart AC, Sippel JL, Wilson GM, Evans CT. Case-fatality with coronavirus disease 2019 (COVID-19) in united states veterans with spinal cord injuries and disorders. Spinal Cord. 2020;58:1040-1.

2. COVID-19 breakthrough Case investigations and reporting. Centers for Disease Control and Prevention. https://www.cdc.gov/vaccines/covid-19/health-departments/ breakthrough-cases.html. Published April 10, 2021. Accessed April 20, 2021.

3. Clinical Spectrum of SARS-CoV-2 Infection Web site. National Institutes of Health. https://www.covid19treatmentguidelines.nih.gov/overview/clinical-spectrum. Accessed 20 April 2021.

4. Teran RA, Walblay KA, Shane EL, Xydis S, Gretsch S, Gagner A. et al. Postvaccination SARS-CoV-2 infections among skilled nursing facility residents and staff members -Chicago, Illinois, December 2020-March 2021. MMWR Morb Mortal Wkly Rep. 2021;70:632-8.

5. Cavanaugh AM, Fortier S, Lewis $\mathrm{P}$, Arora V, Johnson M, George K. et al. COVID-19 outbreak associated with a SARS-CoV-2 R.1 lineage variant in a skilled nursing facility after vaccination program-Kentucky, March 2021. MMWR Morb Mortal Wkly Rep. 2021;70:639-43. https://doi.org/10.15585/mmwr.mm7017e2.

\section{AUTHOR CONTRIBUTIONS}

JLS led the design and build of the registry and operational reports that served as data sources for these analyses, with consultation on registry development provided by $A C E$ and SPB. ACE led development of additional registry reports with data on COVID-19 infection and vaccinations used to identify breakthrough cases. KMS performed the validation chart reviews, and KMS and SPB performed the descriptive analyses. All authors participated in the interpretation of the results. KMS drafted the initial version of the correspondence, and SPB, JLS, and ACE revised the correspondence. All authors approved the final version of the correspondence.

\section{FUNDING}

This work was supported by resources of VA Puget Sound Health Care System, VA St Louis Health Care System, and the VA Spinal Cord Injuries and Disorders National Program Office.

\section{COMPETING INTERESTS}

The authors declare no competing interests.

\section{ETHICS APPROVAL}

This work is based on operations report data and does not constitute a research activity per VHA Program Guide 1200.21. The contents of this work do not represent the views of Veterans Affairs or the United States Government.

\section{ADDITIONAL INFORMATION}

Correspondence and requests for materials should be addressed to K.M.S.

Reprints and permission information is available at http://www.nature.com/ reprints

Publisher's note Springer Nature remains neutral with regard to jurisdictional claims in published maps and institutional affiliations. 